九州大学学術情報リポジトリ

Kyushu University Institutional Repository

A List of the Coleopterous Type Specimens from Chujo-Chujo Collection Donated to Kyushu University, I (Insecta)

Chujo, Michitaka

Chujo, Michio

https://doi.org/10.5109/2607

出版情報: ESAKIA. 37，pp. 39-56，1997-09-30. 九州大学農学部附属彦山生物学実験所 バージョン：

権利関係 : 
ESAKIA, (37): 39-56. September 30, 1997

\title{
A List of the Coleopterous Type Specimens from Chûjô-Chûjô Collection Donated to Kyushu University, I (Insecta)!)
}

\author{
Michitaka CHÛJô \\ Hikosan Biological Laboratory, Faculty of Agriculture \\ Kyushu University, Hikosan, Fukuoka, 824-07 Japan \\ and \\ Michio CĤ̂ Jô \\ Kamata 1-6-16-505, Oota-ku, Tokyo, 144 Japan
}

\begin{abstract}
A list of the holotype specimens, which are personal collections of M. Chûjô and M. T. Chûjô and will be donated to the Entomological Laboratory, Faculty of Agriculture, Kyushu University, is presented. The first list contains 89 species belonging to 59 genera, 3 families in Coleoptera. Each specific epithet has the following 3 items: bibliographical data of original description; transcription of type label; condition of type specimen.

Key words: Coleoptera, holotype list, M. Chûjô and M. T. Chûjô's collection.
\end{abstract}

Holotype specimens preserved by M. Chûjô and M. T. Chûjô as personal collection will be donated to the Entomological Laboratory, Faculty of Agriculture. Kyushu University for good security and domestic preservation. They are consist of coleopterous 3 families, 59 genera and 89 species.

In every species, the original description is shown in "1". "2" shows the transcription of type label put the type specimen, and the condition of the type specimen is described in "3".

\section{CERAMBYCIDAE}

Cylindrecamptus albomaculatus Breuning et Chûjô, 1964

1 Nat. \& Life, S.-E. Asia, 3(31): 249, Pl. 20, Fig. 8a,b. (male)

1) Contribution from the Hikosan Biological Laboratory, Faculty of Agriculture. Kyushu University, Hikosan (Ser. 4, No. 17). 
2 Type label: Cylindrecamptus albomaculatus Breuning et Chûjô, 1964. Khao Chong, Thailand, 4. ii. 1962, K. Yoda leg.

3 Minute pinned. Terminal segment of right and the last 3 segments of left antenna, left front tarsus and the last 2 segments of left middle tarsus are missing.

\section{Exocentrus paraguttulatus Breuning et Chûjô, 1971}

1 Mem. Fac. Educ. Kagawa Univ., 2(202): 31.

2 Type label: Exocentrus paraguttulatus Breuning et Chûjô, 1971 . Yona, Is. Okinawa, 5. ix. 1970, Coll. M. Chûjô.

3 Glued. Very well preserved.

Menesida yoshikawai Breuning et Chûjô, 1968

1 Mem. Fac. Educ. Kagawa Univ., 2(161): 25-26, Fig. 7.

2 Type label: Menesida yoshikawai Breuning et Chûjô, 1967. Cheko, Cambodia, 12. ii. 1965, K. Yoshikawa leg.

3 Glued. Well preserved except for left antenna is missing only apical half of terminal segment.

\section{Mesosa (Aphelocnema) anancyloides Breuning et Chûjô, 1962}

1 Niponius, Acta Col., 1( 16): 2-3, 1 fig.

2 Type label: Mesosa (A phelocnema) anancyloides Breuning et Chûjô, 1962. Chieng Mai, Thailand, 19. v. 1961, K. Iwata leg.

3 Glued. Rather well preserved except for right antenna is missing after 7th segment. Also the last 2 segments of right tarsus off and attached separately to the same card point with the specimen.

\section{Niphona micropuncticollis Breuning et Chûjô, 1961}

1 Nat. \& Life, S.-E. Asia, 1(22): 344-345, Pl. 9, Fig. 6. (female)

2 Type label: Niphona micropuncticollis Breuning et Chûjô, 1961. Chieng Mai, Thailand. 6. iv. 1958, H. Ikoma leg.

3 Pinned. Well preserved.

\section{Phlyarus (Bulbophlyarus) thailandensis Breuning, 1966}

1 Mem. Fac. Educ. Kagawa Univ., 2(135): 24.

2 Type label: Phlyarus (Bulbophlyarus) thailandensis Breuning, 1966. Sampatong, N.

Thailand, 8. xii. 1962, S. Nakao leg. 
3 Glued. Left antenna is missing 2 nd and 3rd segments, and also right antenna after 5th segment, left middle leg except tarsus and right hind leg except femur. Left middle tarsus off and attached to the metasternum and right hind femur also off and attached to the 1st visible abdominal stemite. Left antenna after 3rd segment and the terminal segment of left tarsus off and attached to the same card point with the specimen.

Picibidion laterale nagatomii Villier, 1966

1 Mem. Fac. Educ. Kagawa Univ., 2(135): 20, Fig. 10.

2 Type label: Picibidion laterale nagatomii Villier, 1964. Prew, E. Thailand, 17. i. 1963. A. Nagatomi leg.

3 Glued. Head and prothorax off and attached to the same card point with the specimen. Right antenna has only basal 3 segments remaining. Left antenna missing after 8th segment, and also left hind tarsus.

Planodes ambonensis Breuning et Chûjô, 1965

1 Niponius, Acta Col., 2(9): 51-52, 1 fig. (male)

2 Type label: Planodes ambonensis Breuning et Chûjô, 1965. Salahuti 600m alt., Ambon Is., Indonesia, 24. x. 196 1, A. M. R. Wegner leg.

3 Pinned. Very well preserved.

Pothyne bicolor Breuning et Chûjô, 1961

1 Nat. \& Life, S.-E. Asia, 1(22): 345, Pl. 10, Fig. 10. (female)

2 Type label: Pothyne bicolor Breuning et Chûjô, 1959. Chieng Mai, Thailand. 2 1. v. 1958, H. Ikoma leg.

3 Minute pinned. Left antenna is missing after 6th segment, and also right one after 7th segment.

\section{Pseudotetraglenes cambodgensis Breuning et Chûjô, 1968}

1 Mem. Fac. Educ. Kagawa Univ., 2(161): 23, Fig. 5.

2 Type label: Pseudotetraglenes cambodgensis Breuning et Chûjô. Cheko, Cambodia, 10. ii. 1965, K. Yoshikawa leg.

3 Glued. Left antenna is missing after basal half of 6th segment, also right one after 7th segment, and also right front tarsus after 2nd segment.

Salpinia duffyi Villier, 1966 
1 Mem. Fac. Educ. Kagawa Univ., 2(135):18.

2 Type label: Salpinia dyffyi Villier, 1964. Prew, E. Thailand, 12. i. 1963, A. Nagatomi leg.

3 Glued. Left antenna is missing after 4th segment, and right one after 3rd segment. Left front tarsus missing after 4 th segment, also right front tarsus and left hind leg after femur.

\section{Sybra (Sybra) mimogeminata carinatipennis Breuning et Chûjô, 1970}

1 Mem. Fac. Educ. Kagawa Univ., 2( 192): 56.

2 Type label: Sybra (Sybra) mimogeminata carinatipennis Breuning et Chûjô, 1970. Taketomi, Is. Taketomi, 16. iv. 1969, Coll. M. Chûjô.

3 Glued. Very well preserved.

\section{Sybra (Sybra) musashinoi Breuning et Chûjô, 1970}

1 Mem. Fac. Educ. Kagawa Univ., 2(192): 56.

2 Type label: Sybra (Sybra) musashinoi Breuning et Chûjô, 1970. Is. Uotsuri, Senkaku Is., 10. v. 1969, Coll. R. Musashino.

3 Glued. Well preserved except that left middle leg and left hind tarsus after penultimate segment are missing.

\section{Xylotrechus chujoi Hayashi, 1960}

1 Ent. Rev. Japan, 1 1(1): 24-25.

2 Type label: Xylotrechus chujoi Hayashi, 1960. Yurudji, Is. Okinawa, Loo-choo. 26. vi. 1958, Coll. K. Iha.

3 Glued. Well preserved except that only right tarsus is missing after penultimate segment.

\section{Zorilispe cambodgensis Breuning et Chûjô, 1968}

1 Mem. Fac. Educ. Kagawa Univ., 2(161): 22-23, Fig. 4.

2 Type label: Zorilispe cambodgensis Breuning et Chûjô, 1965. Cheko, Cambodia. 18. ii. 1965, K. Yoshikawa leg.

3 Glued. Left antenna is missing after penultimate segment, also right one after 9th segment, and right elytron.

\section{CHRYSOMELIDAE}

Altica neparensis Chûjô, 1966 
1 Mem. Fac. Educ. Kagawa Univ., 2(145): 29-31, Fig. 1. (male)

2 Type label: Alticaneparensis Chûjô, 1965. Chitare(alt. 2200m), E. Nepal, 24. iv. 1963, K. Yoda leg.

3 Glued. Right middle tarsus is missing after basal segment, and also left hind tibia. Left hind tarsus off but glued on the abdomen and male genitalia removed and mounted on the same card point with the specimen.

\section{Apophilia asahinai Chûjô, 1962}

1 Phil. J. Sci., 91( $1 \&$ 2): 23-26. (male)

2 Type label: Apophilia asahinai Chûjô. Musha, Formosa, 26. x. 1928, Coll. M. Chûjô.

3 Pinned. Right hind tarsus and left hind leg are missing. Elytra and mososternum somewhat damaged by pin.

\section{Apophilia furcigera Chûjô, 1962}

1 Nat. \& Life, S.-E. Asia, 2(24): 102-105, Fig. 1a,b.

2 Type label, male: Apophilia furcigera Chûjô, 1961, Chieng Mai, Thailand, 19. vi. 1958, H. Ikoma leg.

3 Glued. Well preserved except that right antenna after 6th segment off but attached to the same card point with the specimen.

\section{Apophilia securigera Chûjô, 1962}

1 Nat. \& Life, S.-E. Asia, 2(24): 105-106, Fig. 2.

2 Type label, male: Apophilia securigeru Chûjô, 1961. Chieng Mai, Thailand, 19. vi. 1958, H. Ikoma leg.

3 Glued. Right antenna, left front and middle legs are missing. Left antenna after 9th segment removed and mounted on the same card poit with the specimen.

\section{Apteropeda japonica Chûjô, 1951}

1 Trans. Shikoku Ent. Soc., 2(3): 41-42, Fig. 3. (female)

2 Type label: Apteropeda japonica Chûjô. Mt. Tsurugi, Tokushima-ken, Japan. 28. vii. 1949, Coll. M. Chûjô.

3 Glued. Left and right front legs after every femur are missing.

Atysa uetsukii Chûjô, 1954

1 Mushi, 26(1): 2-3, Pl. 1. 
2 Type label: Atysa uetsukii Chûjô. Okayama Pref., Honshu, Japan, 4. viii. 1953, Coll. K. Uetsuki.

3 Minute pinned. Well preserved except for right front leg is missing.

\section{Aulacophora Ioochooensis Chûjô, 1957}

1 Mem. Fac. Educ. Kagawa Univ., 2(52): 2-4. (male)

2 Type label: Aulacophora Zoochooensis Chûjô. Amami-Oshima, 2 1. vii. 1955, Coil. T. S hirôzu.

3 Glued. Well preserved except that right antenna is missing after 9th segment.

\section{Basilepta ikomai Chûjô, 1961}

1 Nat. \& Life, S.-E. Asia, 1(24): 347-348.

2 Type label: Basilepta ikomai Chûjô, 1959. Chieng Mai, Thailand, 14. vi. 1958, H. Ikoma leg.

3 Glued. Well preserved except that antennae are missing after right 3rd segment, and left one after basal segment. Left front leg off and attached to the same card point with the specimen.

\section{Basilepta maebarai Chûjô, 1956}

1 Mem, Fac.Educ. Kagawa Univ., 2(31): 1-3, Fig. 1. (male)

2 Type label: Basilepta maebarai Chûjô. Cape Sata, Kagoshima Pref., Kyushu, 1. viii. 19.55, H. Maebara leg.

3 Glued. Well preserved except for both terminal two segments of antennae off but attached separately to the same card point with the specimen.

\section{Calomicrus iniquus aconiticolus Chûj ô, 1959}

1 Mem. Fac.Educ. Kagawa Univ., 2(81): 10-1 1. (male)

2 Type label: Calomicrus iniquus aconiticolus Chûjô, 1959. Mt. Tsurugi-san. Tokushima Pref., 26. vii. 1954, M. Chûjô leg.

3 Minute pinned. Very well preserved.

Cassida (s. str.) lineola Crertzer var. japonica Chûjô, 1951

1 Trans. Shikoku Ent. Soc.,2(3): 46-47. (male)

2 Type label: Cassida (s. str.) lineola Crertzer. Ikenobe, Kagawa-ken, Japan, 10. v. 1949. Coll. K. Iwata. 
3 Pinned. Well preserved except only right elytron somewhat damaged.

\section{Chaetocnema (Chaetocnema) umesaoi Chûjô, 1961}

1 Nat. \& Life, S.-E. Asia, 1(24): 355-356, Pl. 12, Fig. 9. (female)

2 Type label: Chaetocnema umesaoi Chûjô, 1959. Bangkok, Thailand, 29. xii. 1957. T. Umesao leg.

3 Glued. Well preserved.

\section{Chaetocnema (Tlanoma) tonkinensis taiwanensis Chûjô, 1965}

1 Spec. Bull. Lep. Soc. Japan., (1): 99-100. (female)

2 Type label: Chaetocnema (Tlanoma) tonkinensis tuiwunensis Chûjô, 1962. Musha. C. Formosa, 23. vi. 1961, T. Shirôzu leg.

3 Glued. Left antenna is missing after 2nd segment.

\section{Chlamisus bruneiensis Chûjô, 1964}

1 Nat. \& Life, S.-E. Asia, 3(32): 263-265. (female)

2 Type label: Chlumisus bruneiensis Chûjô, 1962. Brunei, Brunei, 3. iii. 1962, Imadate leg.

3 Glued. Well preserved except for only right front leg off but attached separately to the same card point with the specimen.

\section{Chlamisus imadatei Chûjô, 1964}

1 Nat. \& Life, S.-E. Asia, 3(32): 265-266. (female)

2 Type label: Chlumisus imadatei Chûjô, 1962. Brunei, Brunei, 3. iii. 1962, Imadate leg.

3 Glued. Well preserved except for only left middle tarsus is missing.

\section{Chlamisus shirozui Chûjô, 1956}

1 Tech. Bull. Kagawa Agr. College, 7(1): 155-156, Fig. 15. (male)

2 Type label: Chlumisus shirozui Chûjô. Heigan-Kayo, N. Formosa, 3. vii. 1941. Coll. A. Kira.

3 Minute pinned. Well preserved except that right front leg is missing, and also right middle leg after femur. 
1 Mem. Fac. Educ. Kagawa Univ., 2(47): 4-5, Fig. 2. (male)

2 Type label: Clitea shirahatai Chûjô. Youjyou, Shansi Prov., N. China, 26. v. 1941, Coll. K. Shirahata.

3 Glued. Very well preserved.

\section{Cneorane tibialis Chûjô, 1966}

1 Mem. Fac. Educ. Kagawa Univ., 2( 145): 10-1 1. (male)

2 Type label: Cneorane tibialis Chûjô, 1965. Jumbesi(alt. 2700m), E. Nepal, 27. v. 1963, K. Yoda leg.

3 Glued. Very well preserved.

\section{Coenobius imadatei Chûjô, 1964}

1 Nat. \& Life, S.-E. Asia, 3(32): 262-263.

2 Type label: Coenobius imadatei Chûjô, 1962. Brunei, Brunei, 1. iii. 1962, Imadate leg.

3 Glued. Well preserved.

\section{Colaspoides japana Chûjô, 1956}

1 Mem. Fac.Educ. Kagawa Univ., 2(31): 3-5, Fig. 2.

2 Type label, male: Colaspoides japana Chûjô. Toi-misaki, Cape Sata, Kagoshima Pref.. Kyushu, Japan, 21. v. 1954, Coll. 0. Mizoguchi.

3 Glued. Well preserved except that right middle tarsus and right antenna from 6th to 9th segments off and attached on the other card point pinned under the specimen.

Corynodes satoi Chûjô, 1959

1 Sci. Rep. Agr. Exped. Cambodia, Hyogo Univ. Agr., 2: 56-60. (male)

2 Type label: Corynodes satoi Chûjô, 1958. Kp. Chhnang, Cambodia, 20. iv. 1957. Sat8 \& Takay leg.

3 Pinned. Well preserved except only right hind leg with trochanter is missing.

\section{Cryptocephalus ainu Chûjô, 1959}

1 Mem. Fac. Educ. Kagawa Univ., 2(81): 6-8. (male)

2 Type label: Cryptocephalus ainu Chûjô, 1959. Mt. Tokachi-dake in the range of Mt. Daisetsu-zan, C. Hokkaido, 18. vii. 1954, M. Konishi leg.

3 Minute pinned. Left antenna is missing after 5th segment, and also right one after 4th, left front tarsus, left middle tarsus after 8th, left hind leg after femur, and right leg. 
Demotina elegans Chûjô et Shirozu, 1955

1 Sieboldia, Kyushu Univ., Ent. Publ., 1(3): 239-241. (male)

2 Type label: Demotina elegans Chûjô et Shirozu. Amboo, Is. Yakushima, Kagoshima Pref., Kyushu, Japan, 24. vii. 1950, T. Shirozu leg.

3 Glued. Right middle and hind legs are missing.

Dercetis eurycollis Chûjô, 1965

1 Spec. Bull. Lep. Soc. Japan., (1): 93-95. (female)

2 Type label: Dercetis eurycollis Chûjô, 1962. Tattaka, C. Formosa, 24. vi. 1961. T. Shirôzu leg.

3 Glued. Left antenna after 5th segment off but attached to the same card point with the specimen, and right middle leg is missing. Prothorax and head off but attached to middle thorax.

\section{Dercetis tricolor Chûjô, 1965}

1 Spec. Bull. Lep. Soc. Japan., (1): 95-96.

2 Type label, female: Dercetis tricolor Chûjô, 1962. Rimogan, N. Formosa, 10. vii. 1961, T. Shirozu leg.

3 Glued. Left antenna is missing after 8th segment. Right front leg after femur off but attached to the same card point with the specimen.

\section{Donacia (Donacia) japonica Chûjô et Goecke, 1956}

1 Akitu, Trans. Kyoto Ent. Soc., 5(3): 60-62, 1 fig.

2 Type label, male: Donacia (s. str.) japonica Chûjô et Goecke. Midoro-ga-ike, Kyoto Pref., Honshu, Japan, 7. v. 1951, Coll. T. Kishii.

3 Minute pinned. Well preserved except for only left front tarsus is missing after basal segment.

Editus cupreus Chûjô, 1964

1 Nat. \& Life, S.-E. Asia, 3(32): 271-273. (male)

2 Type label: Editus cupreus Chûjô, 1962. Rambai, Brunei, 10. ii. 1962, Imadate leg.

3 Glued. Well preserved. 
1 Mem. Fac. Educ. Kagawa Univ., 2(52): 5-6. (male)

2 Type label: Epithrix shirozui Chûjô. Amami-Oshima, 21. vii. 1955, Col. T. Shirôzu.

3 Glued. Well preserved.

Galerotella asahinai Chûjô, 1962

1 Phil. J. Sci., 91(1\& 2): 29-33.

2 Type label, male: Galerotella asahinai Chûjô. Tattaka-Musha, 22. vi ii. 11936. no collector's nae.

3 Pinned. Right front leg, and right hind leg are missing after basal half of tibia. Middle part of metastemum somewhat damaged by pin.

\section{Galerucella bruneiensis Chûjô, 1964}

1 Nat. \& Life, S.-E. Asia, 3(32): 290-292, Pl. 26, Fig. 22.

2 Type label: Galerucella bruneiensis Chûjô, 1962. Rambai, Brunei. 12. iii. 1962. Imadate leg.

3 Glued. Right elytron transversely and acutely depressed at about middle part, and left elytron off after $4 / 5$ from base.

\section{Gynandrophthalma gotoi Chûjô, 1957}

1 Mem. Fac. Educ. Kagawa Univ., 2(47): 1-2, Fig. 1 (female)

2 Type label: Gynandrophthalma gotoi Chûjô. Cheng Ching, Hopeh Prov., Manchuria, 21-27. vii. 1940, Coll. M. Gotô.

3 Glued. Both antennae are missing after every 8th segment, and also both tarsus of front legs and right hind tarsus. Both middle and left hind tarsus missing after every 4th segment. Basal 3 segments of right middle tarsus off but attached to the right elytron.

\section{Hamushia konishii Chûjô, 1956}

1 Mem. Fac. Educ. Kagawa Univ., 2(31): 12-14, Fig. 6. (male)

2 Type label: Hamushia konishii Chûjô. Mt. Wakasugi (Chikuzen), Kyushu. 29. iv. 1954, K. Morimoto leg.

3 Glued. Well preserved except that left hind tarsus is missing after basal segment.

\section{Hemipyxis plagioderoides flaviabdominalis Chûjô, 1965}

1 Spec. Bull. Lep. Soc. Japan., (1): 100. (male)

2 Type label: Hemipyxis plagioderoides flaviabdominalis Chûjô, 1962. Tattaka, Formosa, 23. vi. 1961, Col. T. Shirôzu. 
3 Glued. Well preserved except for only left front tarsus is missing after 4th segment. Right middle leg off but attached to the same card point with the specimen.

\section{Hespera aulipilosa loochooana Châjô, 1957}

1 Mem. Fac. Educ. Kagawa Univ., 2(52): 6-7. (male)

2 Type label: Hespera aulipilosa Zoochooanu Chûjô. Amami-Oshima. 28. vii. 1955, Col. T. Shirôzu.

3 Glued. Well preserved.

\section{Hespera formosana albopilosa Chûjô, 1957}

1 Mem. Fac. Educ. Kagawa Univ., 2(52): 7 (male)

2 Type label: Hespera formosana albopilosa Chûjô. Amami-Oshima, 20. vii. 1955. Col. T. Shirôzu.

3 Glued. Well preserved except for only left hind tarsus is missing after basal segment.

\section{Hirtigaleruca aptera Chûj ô, 1962}

1 Phil. J. Sci., 91(1\& 2): 46-47.

2 Type label, male: Hirtigaleruca aptera Chûjô. Tipon, Taito-gun, Taito-Tyo, Formosa. 2. viii. 1936, S. Asahina leg.

3 Minute pinned. Well preserved except that right elytron with a hole each by pin at the postero-medial part and just behind the basal margin.

\section{Hyperaxis ariyani Chûjô, 1964}

1 Nat. \& Life, S.-E. Asia, 3(32): 273-275, Pl. 25, Fig. 15. (male)

2 Type label: Hyperuxis uriyani Chûjô, 1962. Hoy Ceo Valley, Chiengmai, Thailand. 15. v. 1961, K. Iwata leg.

3 Glued. Left front leg is missing after femur, right elytron with a pin hole at middle part.

\section{Lactica thaiensis Chûjô, 1964}

1 Nat. \& Life, S.-E. Asia, 3(32): 303-305.

2 Type label: Lactica thuiensis Chûjô, 1962. In train, Chieng Mai, Thailand, 29. iv. 1961, K. Iwata leg.

3 Glued. Well preserved except for only right elytron with a big pin hole at about middle part. 


\section{Leptispa taguchii Chûjô, 1956}

1 Mem. Fac. Educ. Kagawa Univ., 2(31): 18-20, Fig. 9. (ale)

2 Type label: Leptispa taguchii Chûjô. Kawagoe, Saitama Pref., Honshu, Japan, 13. v. 1938, Coll. M. Taguchi.

3 Glued. Well preserved.

\section{Lipromima minuta (Jacoby) quadrimaculata Chûjô, 1956}

1 Mem. Fac. Educ. Kagawa Univ., 2(31): 17-18, Fig. 8. (female)

2 Typpe label: Lipromima minuta quadrimaculata Chûjô. Mt. Homan-zan, Fukuoka Pref., Kyushu, Japan, 3. viii. 1954, Coll. Y. Tominaga.

3 Glued. Right antenna is missing, also right and left hind tarsi are missing after every 4th segment.

\section{Liroetis neparensis Chûjô, 1966}

1 Mem. Fac. Educ. Kagawa Univ., 2(145): 15-16. (male)

2 Type label: Liroetis neparensis Chûjô, 1965. Chaubas (alt. 2000m), E. Nepal, 20. vi. 1963, K. Yoda leg.

3 Glued. Left leg after femur, right front tarsus after basal segment, right middle tarsus after 2nd segment, left hind tarsus and right hind tarsus are missing. Left middle leg without apical 2 segments and several parts of legs off but attached to the same card point with the specimen.

Luperodes saramao Chûjô, 1962

1 Phil. J. Sci., 91(1\& 2): 230-232. (male)

2 Type label: Luperodes saramao Chûjô. Mururoahu-Kussya (altitude. ca. 1700m). in Mt. Taihei-zan, Taihoku-shu, Formosa, 13. viii. 1936, S. Asahina leg.

3 Glued. Right antenna is missing after 6th segment, and also left middle leg.

\section{Luperomorphella (Luperomorphella) albomaculata Chûjô, 1964}

1 Nat. \& Life, S.-E. Asia, 3(32): 306-307, Pl. 26, Fig. 23. (male)

2 Type label: Luperomorphella (Luperomorphella) albomaculataChûjô. 1962. Prew, Thailand, 17. vi. 1961, K. Iwata leg.

3 Glued. Left middle leg after femur and right middle leg are missing, left hind leg off but attached to the same card point with the specimen. 
1 Mem. Fac.Educ. Kagawa Univ., 2(58): 4-5, Fig. 1. (male)

2 Type label: Lypesthes babai Chûjô, 1957. Sumo, Is. Tsushima, Japan, 27. v. 1957, Coll. K. Baba.

3 Minute pinned. Well preserved except for only right front tarsus is missing terminal segment.

\section{Manobidia nipponica Chûjô, 1959}

1 Mem. Fac. Educ. Kagawa Univ., 2(8 1): 11-13. (male)

2 Type label: Manobidia nipponica Chûjô, 1959. Valley Omogo-kei, Ehime Pref.. 4. v. 1958, M. Chûjô leg.

3 Glued. Well preserved.

\section{Mellipora shirozui Chûjô, 1965}

1 Spec. Bull. Lep. Soc. Japan., (1): 102.

2 Type label: Mellipora shirozui Chûjô, 1962. Oiwake, formosa, 24. vi. 1961, Coll. T. Shirôzu.

3 Glued. Right antenna after 5th segment, right front leg, left front leg after femur and both hind legs off but attached separately to the same card point with the specimen.

Monolepta amiana Chûjô, 1962

1 Phil. J. Sci., 91(1\& 2): 136-137.

2 Type label, female: Monolepta amiana Chûjô. Tyokakurai, Daibu, 26. vii. 1936, no collector' s name.

3 Glued. Well preserved but somewhat depressed.

Monolepta asahinai Chûjô, 1962

1 Phil. J. Sci., 91(1\& 2): 116-1 18. (male)

2 Type label: Monolepta asahinai Chûjô, Heiganzan-Kunugigaoka, Formosa, 18. viii. 1936, Coll. S. Asahina.

3 Glued. Well preserved.

\section{Monolepta Ieperoides Chûjô, 1962}

1 Phil. J. Sci., 91(1\& 2): 126-127.

2 Type label, female: Monolepta Zeperoides Chûjô. Heiganzan-Kunugiga-oka. 18. viii. 1936, no collector's name.

3 Minute pinned. Well preserved. 


\section{Monolepta mandibularis Chûjô, 1962}

1 Phil. J. Sci., 91(1\& 2): 120-123.

2 Type label, male: Monolepta mandibularis Chûjô. Mururoahu-Kussya (altitude, about $1300 \mathrm{~m})$, in Mt. Taiheizan, Taihoku-shu, Formosa, 13. viii. 1936, Coll. S. Asahina.

3 Glued. Very well preserved.

Nodina chalcosoma Baly var. rufofulva Chûjô, 1951

1 Trans Shikoku Ent. Soc.,2(3): 37.

2 Type label: Nodina chalcosoma Baly var. rufofulva Chûjô. Muya-cho, Tokushima-ken, Japan, 21. vi. 1948, Coll. M. Chûjô.

3 Glued. Well preserved.

\section{Nodina parvula thaiensis Chûjô, 1961}

1 Nat. \& Life, S.-E. Asia, 1(24): 349, Pl. 11, Fig. 4. (male)

2 Type label: Nodina parvula thaiensis Chûjô, 1959. Chieng Mai, Thailand, 9. v. 1955. H. Ikoma leg.

3 Glued. Right antenna after 7th segment off but attached separately to the same card point with the specimen.

\section{Oulema (Gracilema) iwatai Chûjô, 1964}

1 Nat. \& Life, S.-E. Asia, 3(32): 257-258, Pl. 24, Fig. 9.

2 Type label: Oulema (Gracilema) iwatai Chûjô. Chiengmai, Thailand, 29. iv. 1961, K. Iwata leg.

3 Glued. Right front and left hind legs are missing.

\section{Parachalcoides babai Chûjô, 1959}

1 Mem. Fac. Educ. Kagawa Univ., 2(81): 14-15. (male)

2 Type label: Parachalcoides babai Chûjô, 1959. Tappi, Higashi-tsugaru-gun, Aomori Pref., 9. viii. 1956, K. Baba leg.

3 Glued. Well preserved except for only left front tarsus is missing apical half of terminal segment.

\section{Phaedon yodai Chûjô, 1966}

1 J. College Arts \& Sci., Chiba Univ., 4(4): 553-554. 
2 Type label: Phaedon yodai Chûjô, 1965. Jumbesi 3280m, E. Nepal, 22. v. 1963, K. Yoda leg.

3 Glued. Left antenna is missing after 1st segment, and also right middle leg.

Philopona vibex (Erichson) var. nigra Chûjô, 1951

1 Trans. Shikoku Ent. Soc., 2(3): 44. (male)

2 Type label: Philopona vibex var. nigra Chûjô. Muya-cho, Tokushima-ken, Japan. 29. iv. 1949, Coll. M. Chûjô.

3 Minute pinned. Very well preserved.

\section{Pseudometaxis maculatus Chûjô, 1964}

1 Nat. \& Life, S.-E. Asia, 3(32): 279-280, Pl. 25, Fig. 16.

2 Type label: Pseudometaxis maculatus Chûjô, 1962. Hoi Kao Valley, Chiengmai. Thailand, 15. v. 1961, K. Iwata leg.

3 Glued. Well preserved except that right antenna are missing after 10th segment, and left one after 8th.

\section{Psylliodes angusticollis rishiriensis Chûjô, 1959}

1 Mem. Fac. Educ. Kagawa Univ., 2(81): 15-16. (male)

2 Type label: Psylliodes angusticollis rishiriensis Chûjô, 1959. Is. Rishiri-to, an adjacent island of the northern part of Hokkaido, 1. viii. 1953, M. Suda leg.

3 Glued. Well preserved except for only left hind tarsus is missing after 2 nd segment.

Thailandia chakratongii Chûjô, 1964

1 Nat. \& Life, S.-E. Asia, 3(32): 283.

2 Type label, male: Thailandia chakratongii Chûjô, 1962. Doi Suthep, Chiengmai.

Thailand, 19. v. 1961, K. Iwata leg.

3 Glued. Well preserved.

Zeugophora (Pedrillia) annulata (Baly) forma inannulata Chûjô, 1959

1 Mem. Fac. Educ. Kagawa Univ., 2(8 1): 1. (female)

2 Type label: Zeugophora (Pedrillia) annulata f. inannulata Chûjô, 1959. Mt. Ozakiyama, Hiraka-cho, Minamitsugaru-gun, Aomori Pref., 4. iv. 1940, K. Shimoyama leg.

3 Glued. Every last 2 segments of left front and right hind tarsi are missing. 


\section{CURCURIONIDAE}

Caenosilapilus babai Chûjô et Morimoto, 1959

1 Kontyû, 27(2); 148-150, Fig. 2A-E.

2 Type label, male: Caenosilapilus babai Chûjô et Morimoto, 1959. Miyahira. Niigata Pref., 3. vi. 1955, K. Baba leg.

3 Glued. Three basal segments of left antenna remaining, terminal segment of front tarsus, right middle tarsus, left hind leg and terminal segment of right hind tarsus are midding. Left middle and right hind legs off but attached separately to the same card point with the specimen.

Coeliodes babai Voss et Chûjô, 1960

1 Niponius, Acta Col., 1(3): 1-3.

2 Type label, male: Coeliodes babai Voss et Chûjô. Kurokawa, Niigata Pref.. Honshu, Japan, 9. vi. 1955, Coll. K. Baba.

3 Glued. Well preserved.

\section{Elleschus bicoloripes Voss et Chûjô, 1960}

1 Niponius, Acta Col., 1(3): 9-10. (= Adorytomus bicoloripes Voss et Chûjô, female)

2 Type label: Elleschus bicoloripes Voss et Chûjô, 1959. Kamiishikawa, Niigata Pref., Honshu, Japan., 5. v. 1956, Coll. H. Koike.

3 Glued. Head off but attached separately to the same card point with the specimen, right middle tarsus is missing terminal segment, and also right hind tarsus after basal segment.

\section{Elleschus pauper Voss, 1960}

1 Mem. Fac. Educ. Kagawa Univ., 2(94): 9-10.

2 Type label: Elleschus pauper Voss, 1958. Omogo, Ehime, Japan, 4. v. 1958. Coll. M. Chûjô.

3 Glued. Well preserved.

Hylobius vossi Chûjô, 1959

1 Niponius, Acta Col., 1( 1): 1-3, 1 fig.

2 Type label, male: Hylobius vossi Chûjô, 1959. Cape Sata, Kagoshima, 3. v. 1955. H. Fukuda leg.

3 Glued. Right antenna is missing after 2nd segment, and also left middle tarsus. right hind tarsus after 4th segent. 


\section{Isonycholips gotoi Voss et Chûjô, 1960}

1 Mem. Fac. Educ. Kagawa Univ., 2(94): 14-15, Abb. 1.

2 Type label: (gen. et sp.): Isonycholips gotoi Chûjô et Voss, 1959. Akogigaura, Mie Pref., Honshu, Japan, 3. vi. 1956, Coll. M. Gotô.

3 Glued. Well preserved except that only left and right hind tarsi are midding every terminal segment.

Keibaris babai Chûjô, 1960

1 Niponius, Acta Col., 1(5): 1-2, 1 fig. (male)

2 Type label: Keibaris babai Chûjô, 1960. Uchiyama, Is. Tsushima, Japan, 26. v. 1957. Coll. K. Baba.

3 Glued. Well preserved except for only right antenna is missing after 5th segment.

Limnobaris babai Chûjô et Morimoto, 1959

1 Kontyû,27(2): 153-154, Fig. 5.

2 Type label, male: Limnobaris babai Chûjô et Morimoto, 1959. Kurokawa, Niigata Pref., Honshu, Japan, 8. v. 1955, Coll. K. Baba.

3 Glued. Well preserved except for only left middle tarsus and terminal segment of right tarsus are missing.

\section{Metapocyrtus (Trachycyrtus) yonagunianus Chûjô, 1971}

1 Mem. Fac. Educ. Kagawa Univ., 2(202): 35-36. (male)

2 Type label: Metapocyrtus (Trachycyrtus) yonagunianus Chûjô, 197 1. Yonaguni I., Ryukyus, 12. vi. 1965, K. Iha leg.

3 Glued. Well preserved except that left antenna is missing after 4th segment, and also both terminal segments of hind tarsi.

Paracalendra saccharivora Chûjô et Morimoto, 1959

1 Akitu, Trans. Kyoto Ent. Soc., 8(2): 26-29, Fig. 1 \& 2A-E. (male)

2 Type label: Paracalendra saccharivora Chûjô et Morimoto, 1959. Is. Okinawa, Loochoo, 25. xii. 1958, Coll. T. Lora.

3 Glued. Well preserved.

Rhadinomerus maebarai Chûjô et Voss, 1960

1 Mem. Fac. Educ. Kagawa Univ., 2(94): 6. 
2 Type label: Rhadinomerus maebarai Chûjô et Voss, 1960. Mt. Takachiho, Kirishima, 5. vi. 1955, H. Maebara leg.

3 Glued. Well preserved except that right middle tarsus is missing only terminal segment.

\section{Sphinxioides koikei Voss et Chûjô, 1960}

1 Niponius, Acta Col., 1(3): 7-9, Fig. 1. (= Elleschus dissimilis Voss et Chûjô, female) 2 Type label, male: Sphinxioides koikei Voss et Chûjô, 1959. Kami-Ishikawa. Niigata. Japan, 5. v. 1956, Coll. H. Koike.

3 Glued. Well preserved except that right hind tarsus is missing only terminal segment.

Tachypterellus dorsalis Voss et Chûjô, 1960

1 Niponius, Acta Col., 1(3): 4-6.

2 Type label, male: Tachypterellus dorsalis Voss et Chûjô. Mt. Yakemine. Niigata Pref., Honshu, Japan, 19. vii. 1949, Coll. H. Koike.

3 Glued. Well preserved. 